\title{
Methodology approaches and challenges in population-based longitudinal study of a neuroprotective model for healthy longevity
}

\begin{abstract}
Aim: The 36-month Long-Term Research Grant Scheme project: Towards Usual Aging Neuroprotective Model for Healthy Longevity among Malaysian Elderly was designed to address multidimensional aspects including psychosocial, biophysical health, nutrition and dietary pattern, and auditory and visual function to highlight the magnitude of these associations in a single study.

Methods: A total of 2322 respondents aged $\geq 60$ years were recruited at baseline using the multistage sampling method, followed up at 18 months and 36 months.

Results: Response rates at baseline, 18 months and 36 months were 87.8\%, 77.3\% and $67.1 \%$, respectively. At baseline, the prevalence of successful aging, usual aging and mild cognitive impairment was $11 \%, 73 \%$ and $16 \%$, respectively. The prevalence of single and multimorbidity at baseline were $25.9 \%$ and $50.3 \%$, respectively. The incidence rates of mild cognitive impairment at 18 months and 36 months were 6.5 and 5.6 per 100 person-years. The incidence rates of multimorbidity at 18 months and 36 months were 23.7 and 21.5 per 100 person-years, respectively.

Conclusions: The Long-Term Research Grant Scheme project: Towards Usual Aging study provides an opportunity to investigate the interactions between wide ranges of aspects of the older population in a nationally representative sample of the older population.
\end{abstract}

Keyword: Healthy aging; Longitudinal; Mild cognitive impairment; Morbidity; Successful aging 